\title{
Simulation of Vacuum Assisted Resin Infusion (VARI) process for the production of composite material parts
}

\author{
Joana M. Malheiro* \\ Composite Research Group, PIEP - Innovation in Polymer Engineering \\ University of Minho, Campus de Azurém, 4800-058 Guimarães, Portugal \\ Email: joana.malheiro@piep.pt
}

J. P. Nunes

Institute of Polymers and Composites, University of Minho,

Campus de Azurém, 4800-058 Guimarães, Portugal

Email:jpn@dep.uminho.pt

\begin{abstract}
Summary
The Vacuum Assisted Resin Infusion (VARI) is manufacturing process used worldwide to produce composite parts having great diversity of dimensions (from small to very large ones) and geometrical complexity. This manufacturing process is particularly versatile, to produce small series of high performance structural parts. In these cases, the simulations of the VARI process is a very useful tool to define the infusion strategy and to plan and predict the resin flow progress in order to reduce the material waste and manufacturing cycle time and obtaining lighter structures, having lower void fraction and higher fibre content and mechanical performance. The numerical simulation of the VARI process implies the modelling of different complex phenomena, such as flow in porous media, mechanical deformation, heat exchange and chemical reaction. Therefore, a finite element software was used to solve a combination of governing equations based on a combination of pre-defined theoretical assumptions, by considering a moving mesh and appropriated boundary conditions. In this work, results obtained from simulations of VARI process were used to define the best strategy to be applied in the production of composite parts with different geometries, sizes and materials and predict the possibility of defects occur. In order to validate the accuracy of simulations, the numerical results were compared with those experimental ones obtained from the production of different composite parts where the best processing strategies were implemented. After analysing and discussing the theoretical and experimental obtained results, changes were applied to the numerical model to improve simulation accuracy.
\end{abstract}

Keywords: composite materials, VARI, composite material manufacturing simulation, finite element, porous medium flow, material characterization.

\section{Introduction}

The increase of production rates and from quality of manufactured parts is implying a growing interest by composite manufacturing process simulation and by its stronger predictive accuracy ${ }^{[1]}$. Among the industrial manufacturing processes used, resin infusion appears more and more as the best economical alternative to manufacture large and/or high mechanical performance parts, with large fibre fractions (wind turbine blades, structural components, wings of aircrafts, etc.). Composite manufacturing processes by resin infusion have been developed for years to bring a cheaper solution to big parts production. Those processes allow a significant cost reduction in raw materials storage and mould fabrication, shorter cycle times, less void formation and avoid the use of trial and error time-consuming procedures to optimise the process parameters ${ }^{[3]}$. In recent years, the interest in using out-of-autoclave (OOA) processing techniques, such as resin infusion ones, has also been renewed because of the very expensive initial investment required by the traditional methods in autoclave, particularly when the composite parts and structures to be produce become larger and more complex.
Furthermore, the possibility of simulating these liquid resin infusion processes allows to predict potential defects on the final parts and reduce significantly the time for setting and optimising their processing parameters ${ }^{[2]}$. In fact, without using computer simulations, the success of these resin infusion methods become highly dependent upon operator skill and experience, particularly in the development of new manufacturing strategies for complex parts. Process modelling, as a predictive computational tool, aims to address and improving the reliability and waste issues that usually result from traditional trial-and-error approaches. Basic modelling attempts generally focus on simulating fluid flow through an isotropic porous reinforcement material. Currently, the more recently developed advanced numerical algorithms are also beginning to take into account the multiscale and multidisciplinary complexity of the reinforcement materials, increasing the accuracy of the simulations ${ }^{[4]}$. In the case of resin infusion manufacturing with textile reinforcement materials, the physical draping of the fabric and the subsequent resin flow through the material are the key stages of the process [4].

In this paper, the accuracy of the results obtained by the simulation of Vacuum Assisted Resin Infusion (VARI) 
manufacturing process is assessed. To accomplish that, the simulation of the infusion of composite parts with different geometries (from a planar plate to a hull of a boat) was performed and compared to the experimental results, obtained from the actual production of the same components. The main limitations of the numerical model are pointed out as well as the considerations and assumptions necessary to obtain more accurate numerical results.

\section{Vacuum Assisted Resin Infusion (VARI)}

The Liquid Composite Moulding (LCM) is a generic term for a family of related processes in composites manufacturing, in which continuous fibres used as reinforcement are first placed in a mould, then a polymer matrix (usually thermosetting) is injected as liquid resin in the cavity ${ }^{[5]}$. The Vacuum Assisted Resin Infusion (VARI), in particular, consists in impregnating a dry preform placed onto a rigid halfmould and under a vacuum bag ${ }^{[1]}$. Then, the pressure differential between a vent pipe connected to a vacuum pump (aprox. at 0 bar) and the injection line (at atmospheric pressure) induces the infusion of the resin along and across the reinforcement. After complete impregnation, the part is subjected to a curing process, usually out of autoclave. For the infusion, several injection ports, injection lines or a tree of injection channels can be used. It is necessary to select a good configuration of injection ports and vents to avoid dry spots and minimize filling time. The VARI process eliminates the costs associated with matched metal tooling, reduces volatiles emission and allows the use of lower resin injection pressures. Also enables the use of low-cost tooling while still producing high quality composite parts with complex geometries ${ }^{[6]}$. Despite the versatility of the VARI process, the reliability and repeatability issues still is, however, a concern for the widespread adoption of this manufacturing process by the advanced industry, when well-validated simulations are not used. The efforts to simulate the resin infusion manufacturing process aim to address the reliability and repeatability concerns in a cost-effective manner ${ }^{[4]}$. Numerical simulations of mould filling can be of great help to avoid problems such as resin rich areas, air bubbles, dry spots, zones of high porosity, as well as the formation of cracks following cure shrinkage. It is also advantageous to determine the optimal infusion strategy ${ }^{[5][6]}$.

\section{Governing Equations}

The resin infusion process is particularly complex to model. In general, the manufacturing process is divided into four main phenomena [4][5]: the physical accommodation of the reinforcement material lay-up to the mould (draping); the flow of the resin through the reinforcement material (infusion); the exothermal reaction of the resin (curing), consequent thermal analysis of heat exchange between the part, mould and environment and the influence of all these factors on the resin viscosity ${ }^{[4]}$.

Up until a few years ago, many flow models that were still used by industry lacked enough precision, because they relied on the assumptions of a homogenous, continuum-based approximation of the preform domain and neglect through- thickness effects, saturation, compaction, and heat transfer. Recently, finite-element based methods have been developed with increasing sophistication, to take into account not only the interdependence of the different phenomena that influences the infusion process but also almost all factors that affect resin flow behaviour. These last factors are: permeability, pressure, viscosity, temperature and heat exchange, variability and susceptibility to handling and cutting of reinforcement, presence of passive apparatus (such as inlets, outlets, flow enhancing materials, etc.), through-thickness effects (effect negligible in thin composite parts), deformation-dependent permeability properties, saturation, tool compaction (because the process uses a flexible film semi-tooling, which deforms simultaneously under the internal mould depression and in result of resin infiltration), void formation, among others ${ }^{[5]}$.

The main phenomena and respective mathematical models usually considered in infusion process simulations are summarized in Table 1.

Table 1- Governing phonomena and mathematical models used in infusion process simulations ${ }^{[5]}$.

\begin{tabular}{|c|c|c|}
\hline & Phenomena & Mathematical model \\
\hline 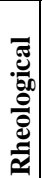 & $\begin{array}{l}\text { Resin flow in a porous } \\
\text { medium } \\
\text { Variation of viscosity }\end{array}$ & $\begin{array}{c}\text { Darcy's law } \\
\text { Constitutive law }\end{array}$ \\
\hline : & $\begin{array}{l}\text { Mould: conduction, loss in } \\
\text { surface } \\
\text { Part: conduction, } \\
\text { convection, generation of heat, } \\
\text { superficial heat loss }\end{array}$ & $\begin{array}{c}\text { Heat equation, transfer } \\
\text { coefficient (convection-radiance) } \\
\text { Equation of convection- } \\
\text { diffusion with source term, model } \\
\text { with one temperature }\end{array}$ \\
\hline |ֶ.ّ. & $\begin{array}{l}\text { Transport of chemical } \\
\text { species, diffusion, } \\
\text { polymerization }\end{array}$ & $\begin{array}{l}\text { Equation of convection- } \\
\text { diffusion with source term, } \\
\text { kinetic model (Kamal-Sourour) }\end{array}$ \\
\hline 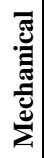 & Mould deformation & $\begin{array}{l}\text { Newtonian's law } \\
\text { Empirical models }\end{array}$ \\
\hline
\end{tabular}

\subsection{Flow in porous media}

In the VARI process, the resin flows through a fibrous reinforcement, which can be considered as a porous medium. In this case, the flow of resin is governed by Darcy's Law, which states that the flow rate of resin per unit area is proportional to the pressure gradient and inversely proportional to the viscosity of the resin. The constant of proportionality is the permeability of the porous medium. It is independent from the fluid, but it depends on the direction of the fibres in each layer of reinforcement. Also capillary forces of attraction or repulsion, which depend on the resin surface tension and its ability to adhere to the surface of fibres and that may also affecting the forehead of flow, by either reducing or increasing 
the effective pressure at the resin front. However, these latter effects are generally considered too small and, therefore, neglected by almost all numerical models. So, assuming that the resin is an incompressible fluid (generalized Newtonian fluid) that travels at low velocity trough a porous medium and the permeability of the porous media is $10^{-3} \mathrm{~m}^{2}$ or less, the Darcy's Law may be written as ${ }^{[4]}{ }^{[7]}$ :

$$
\vec{V}=-\frac{K}{\mu} \vec{\nabla} P
$$

where, $K$ is the permeability tensor, $\mu$ is the viscosity of the resin, $V$ is the Darcy's velocity and $P$ is the pressure (overall pressure gradient through the system) ${ }^{[4]}$.

The permeability characterizes the relative facility that a viscous liquid has in flowing through a porous medium in order to impregnate it. This physical property of the porous medium (cloth, fabric, fibre mat, etc.) depends on the fibre volume fraction (degree of compaction), orientation and configuration of fibres and draping of plies. The permeability of the reinforcement in their principal directions may be determined experimentally.

\subsection{Draping (Mechanical properties)}

From a mechanical perspective, draping behaviour has proven to be difficult to replicate accurately. Woven warp and weft yarns exhibit considerable tensile strength and stiffness but are highly susceptible to reorientation under shear and bending modes. Therefore, any attempt to model draping must accurately account for the yarn reorientation that result from shear loading ${ }^{[4]}$. In order to replicate the mechanical behaviour the characterization of the reinforcement tensile, shear, and bending properties is mandatory.

\subsection{Thermal Phenomena}

The final impregnated part that will lie in the cavity of the mould, consists of reinforcements and resin, which first fills the mould and then becomes progressively polymerized. Heat transfer phenomena significantly affect mould filling and resin curing. Indeed, the temperature of the resin governs the reactivity of the polymerization reaction. Temperature also has an influence on mould filling, since the viscosity of the resin depends on temperature. Thermal simulation are therefore delicate to conduct because of all the related phenomena. Firstly, heat is transferred by conduction between the fibres and the resin. Secondly, a convective transport of heat occurs during the filling of the cavity by the resin. Finally, heat is produced by the exothermic chemical reaction of resin polymerization. Some heat is also created by the viscous dissipation during the resin flow, but in lower degree than the heat originated by the chemical reaction of cure. The temperature field is governed by the general equation:

$$
\rho C_{p} \frac{\partial T}{\partial t}+\rho_{r} c_{p r} \vec{V} \square \nabla T=\vec{\nabla}\{k \square \nabla T\}-\rho_{r} \Delta h \frac{D \alpha}{D t}
$$

where $T$ denotes temperature, $t$ is the time, $\rho$ is the density, $C_{p}$ is the specific heat, $k$ is the heat conduction coefficient tensor, the subscript $r$ designates the resin, $\Delta h$ is the total enthalpy of the cure reaction of the resin, $\alpha$ is the degree of resin cure conversion. This general equation enables to treat the steps of pre-heating, filling and curing.

\subsection{Viscosity of the resin}

The viscosity of the resin depends highly on the temperature and degree of cure conversion, by assuming that viscosity will be infinite when the resin reaches gelation. The dependence of viscosity on these factors can be modelled by a range of different assumption and respective constitutive laws, such as: constant viscosity (Newtonian fluid); predefined law considering the viscosity dependence on temperature; predefined law considering the viscosity as function of temperature and resin curing rate; predefined law considering the viscosity as a function of temperature and resin strain and curing rate.

\subsection{Kinetics of resin polymerization}

The kinetics of polymerization of the resin is usually simulated by the model of Kamal-Sourour, and is essential to describe the curing reaction of the resin ${ }^{[5]}$. In this study, the effect of the resin polymerization will be neglected because the gel time of the resin is assumed to be sufficiently long for not affecting the resin viscosity, which is made constant, and that the curing reaction will take place long time after the infusion process is finished.

\section{$4 \quad$ Numerical method}

In this study, the PAM-RTM ${ }^{\circledR}$ finite element software from ESI was used to simulate the infusion process. It is based on the coupling between the resin flow, governed by Darcy's law, and the preform behaviour considered as porous medium undergoing deformations accordingly to the Terzaghi's principle. The numerical algorithm also considers the changing thickness of the laminate and compaction as a function of the fibre volume content during the infusion ${ }^{[1][5][7]}$. For that, the software decomposes space and time, being the system divided in three zones in space ${ }^{[2]}$ : Stokes zone (fast flow zone constituted by the distribution medium and the resin); Darcy zone (incompressible flow of the resin in the preforms submitted to finite deformations); and dry preforms zone (zone constituted of non-impregnated preforms submitted to finite strains). On the other hand, time is divided in four periods that correspond to the following changes in boundary conditions or physical problem ${ }^{[2]}$ : pre-filling (initial compaction of the preforms due to the vacuuming of the system); filling; postfilling (re-compaction or "rest period" ending by the mechanical equilibrium mandatory to the dimensional quality of the final part); and curing. The model also take into account the porous medium deformation during the temperature and pressure cycles, and deals with the influence of the preform deformation on permeability, and therefore on pressure distribution. Moreover, a thermo-chemical model describes viscosity changes during the infusion ${ }^{[7]}$. More details of the algorithm used can be found in the work of Celle et al ${ }^{[7]}$ and Dereims et al. ${ }^{[9]}$. The software allows Dirichlet or Neuman boundary conditions, and takes into account the effect of 
gravity, which is important in large structures and negligible in small parts ${ }^{[5]}$.

\section{$5 \quad$ Results and Discussions}

The results of the infusion process to manufacture different parts in composite materials are presented below, where the simulation results are compared to the experimental results. For simulations, it was necessary to characterize properly both resin and reinforcement materials.

The permeability of the reinforcement materials, and its variation of the combining effects of orientation and configuration of fibres, draping of plies, compression, etc, is difficult to measure accurately, but its determination is paramount in the simulation of the VARI process. To overcome this problem, a methodology to determine and validate numerically this parameter is presented. After numerically validate the experimental parameters, the simulation of different composite parts are performed and experimentally verified. To accomplish that, simple geometries with simple laminates are firstly validated, then the same is done for geometries and laminates increasingly complex. In the process different assumptions and simplifications are admitted without compromising the accuracy of the numerical results.

\subsection{Flat square Plates}

A Brookfield viscometer was used to measure the viscosity of a the polyester resin Distriton 3501S with $1.5 \%$ of hardener (NOROX MCP) along time. Table 2 summarises the results obtained from those tests. The resin behaves as a Newtonian fluid, with constant viscosity of $469 \mathrm{mPa} . \mathrm{s}$, and allows, approximately, 150 minutes of working time. The long gel time allows to perform the infusion without significant variations in viscosity, and ensuring that the curing process takes place after the complete impregnation of the laminate.

Table 2- Resin Properties - Polyester resin Distitron $3501 \mathrm{~S}$.

\begin{tabular}{|c|c|c|c|c|}
\hline $\begin{array}{c}\text { Density } \\
\left(\mathrm{g} / \mathrm{cm}^{3}\right)\end{array}$ & $\begin{array}{c}\text { Viscosity } \\
(\mathrm{mPa} . \mathrm{s})\end{array}$ & $\begin{array}{c}\text { Gel } \\
\text { Time } \\
(\mathrm{min})\end{array}$ & $\begin{array}{c}\text { Curing } \\
\text { time } \\
(\mathrm{min})\end{array}$ & $\begin{array}{c}\text { Exothermic } \\
\text { Peak } \\
\left({ }^{\circ} \mathrm{C}\right)\end{array}$ \\
\hline 1.12 & 469 & $\approx 150$ & $22-32$ & $140-170$ \\
\hline \multicolumn{5}{c|}{ Curing Cycle } \\
\hline
\end{tabular}

The reinforcement properties are presented in Table 3. A glass fibre unidirectional stitched fabric was used as reinforcement of a laminate of $300 \times 300 \mathrm{~mm}$, which had only one reinforced layer. The permeability along the two main directions of the glass fibre fabric is, respectively, $K_{l}=1.090 \mathrm{E}-$ $08 \mathrm{~m}^{2}$ and $K_{2}=1.250 \mathrm{E}-10 \mathrm{~m}^{2}$, assuming that gravity and thickness of the laminate have no effect.

Table 3- Reinforcement Properties - Glass fibre fabric

\begin{tabular}{|c|c|c|c|c|}
\hline $\begin{array}{c}\text { Density } \\
\left(\mathrm{kg} / \mathrm{m}^{3}\right)\end{array}$ & Structure & $\begin{array}{c}\text { Weight } \\
\left(\mathrm{g} / \mathrm{m}^{2}\right)\end{array}$ & $\begin{array}{c}\text { Orientation } \\
\left({ }^{\circ}\right)\end{array}$ & $\begin{array}{c}\text { Thickness } \\
(\mathrm{mm})\end{array}$ \\
\hline 2600 & $\begin{array}{c}\text { Unidirectional, } \\
\text { stitched }\end{array}$ & 300 & $0^{\circ}$ & 1.0 \\
\hline
\end{tabular}

Figure 1 and Figure 2 compare, at the same moment in time, the experimental and numerical results obtained when two different types of arrangements were used for the resin inlet and outlet (with and without runners). As may be seen, good agreement between the numerical and experimental results is observed. As Figure 3 shows, the resin flow front and the filling time depends on the type of resin inlet. As this last figure depicts, the filling time is lower when runners are used as resin inlet $(\mathrm{t}=1515 \mathrm{~s})$ than without runners $(\mathrm{t}=6520 \mathrm{~s})$, while experimentally the infusion took the similar values of, approximately, 1476 and 6180 seconds, respectively. For both situations, simulations predicted that a volume of resin of approximately $4.60 \mathrm{E}-05 \mathrm{~m}^{3}$ will be used in the infusion, while experimentally a volume around $5.00 \mathrm{E}-05 \mathrm{~m}^{3}$ was used.

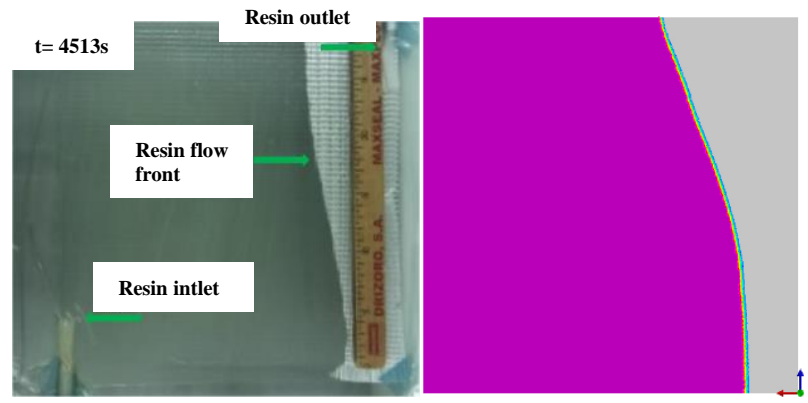

Figure 1- Infusion of a plane laminate without runners at $\mathrm{t}=4513 \mathrm{~s}$ : experimental (left); simulation (right)

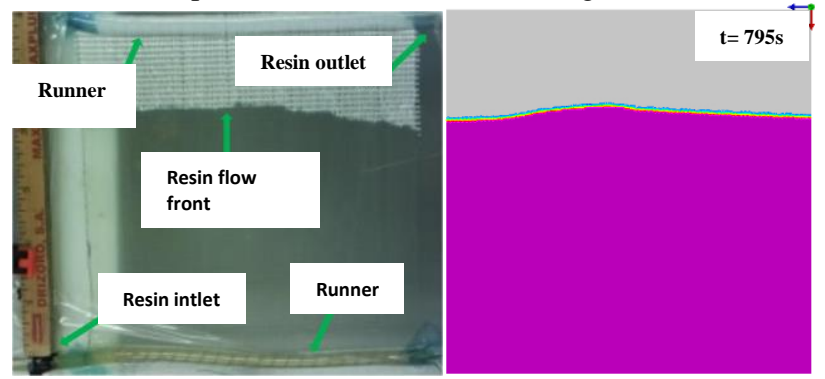

Figure 2- Infusion of a plane laminate with runners at $\mathrm{t}=795 \mathrm{~s}$ : experimental (left); simulation (right).
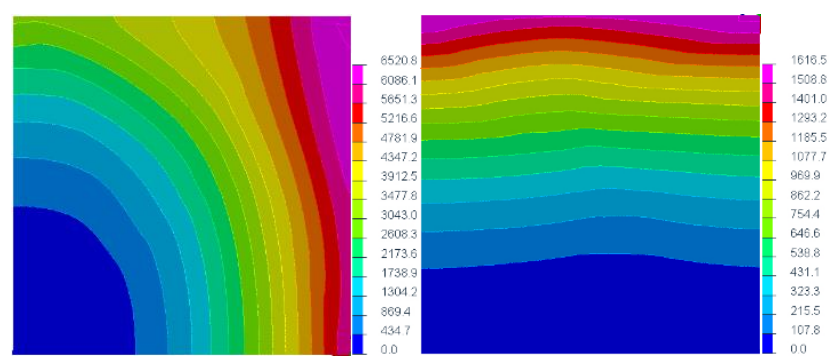

Figure 3 - Filling time for infusion with (right) an without (left) runners.

\subsection{Guitar plate}

The same kind of study was performed for a plate with a complex geometry, namely, a pickguard of a guitar. In this case, the laminate had five layers of carbon fibre fabric with the following properties: plain $\left(0^{\circ} / 90^{\circ}\right)$; weight per unit area: $195 \mathrm{~g} / \mathrm{m}^{2}$; density: $1770 \mathrm{~kg} / \mathrm{m}^{3}$; thickness: $0.30 \mathrm{~mm}$. The single layer of reinforcement presents the following permeability along its main directions: $K_{l}=K_{2}=8.304 \mathrm{E}-11 \mathrm{~m}^{2}$. As matrix was 
used a polyester resin, with the following properties: viscosity: 0.300 Pa.s; density: $1200 \mathrm{~kg} / \mathrm{m}^{3}$. The properties of the resin were considered constant in time.

Although, this part is actually a flat plate, it presents a complex boundary geometry, with different curves and cuts. These characteristics will affect the resin flow front during impregnation because resin flows faster along the reinforcement boundaries. This was observed independently of the type of arrangement used for the resin inlet and outlet, and partially explains the difference between the experimental and numerical results (Figure 4 and Figure 5 a) and b)). Thus, by changing the model and assuming that the permeability was higher at the reinforcement boundaries, both experimental and numerical results start to approximate as it may be seen in Figure 5 a) and c). Still, differences between numerical and experimental resin front outline obtained (Figure 5) were significant. This is easily explained by the permeability admitted in the simulation, which was determined experimentally for one single layer (following the same methodology of the previous example) while the laminate is a stack of five layers, i.e., effects, for example, of draping and compression were not taken into account in the global permeability of the laminate.
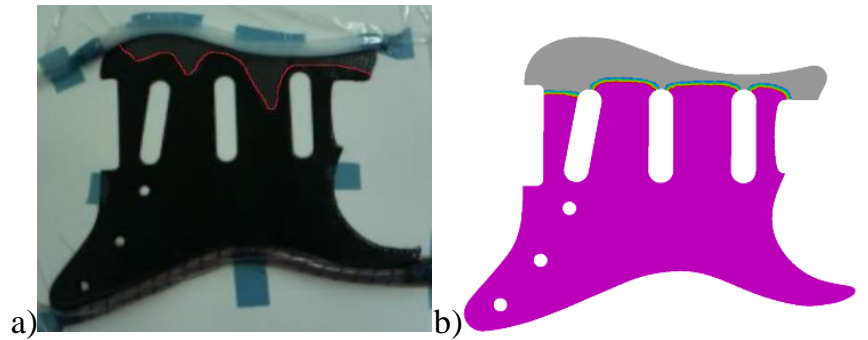

Figure 4- Flow front at $t=2040$ s: experimental (a), simulation (b).

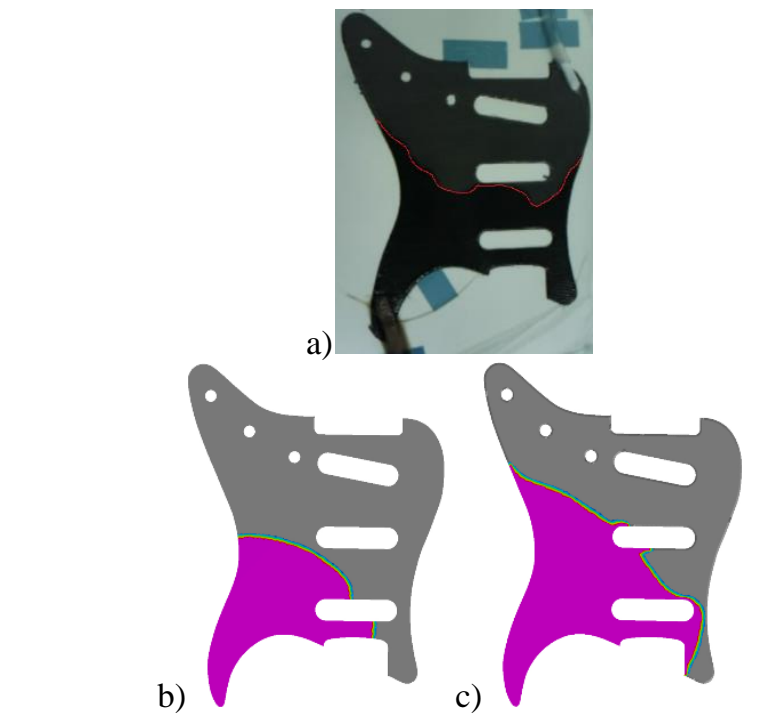

Figure 5- Resin flow front at $\mathrm{t}=1840 \mathrm{~s}$ : experimental (a), simulation (b), simulation with different $K$ at boundaries (c).

\subsection{Car seat part}

The assumptions admitted previously were applied in the production of a component from a car seat (Figure 7-a). The laminate was manufactured by using the same carbon fibre fabric, but twice as many layers (10 layers) than those used in the guitar plate. Since a different laminate is used, it will present necessarily a distinct permeability. Knowing, from the previous example, that the software does not allow to get an accurate simulation of the real resin flow front advance by using the permeability of the single elemental layer used, the permeability of the ten layer laminate was determined experimentally and validated numerically as previously described in point 5.1 and Figure 2. In order to quantify the new permeability, the validation of the infusion of the laminate was performed (Figure 6), using an epoxy resin (density:1140 $\mathrm{kg} / \mathrm{m}^{3}$ ) which behaves as a Newtonian fluid during the infusion, with constant viscosity equal to 0.170 Pa.s. A permeability of $K_{l}=K_{2}=6.827 \mathrm{E}-12 \mathrm{~m}^{2}$ was determined for the laminate by using this procedure (Figure 6-a). Such permeability is considered as the global permeability of the laminate, which means the effect of compressibility and draping between layers, that are difficult to quantify and mimic in the simulation, are accounted for. Thus, the following simplifications were assumed to validate the permeability in the simulation: i) the geometry of the laminate is a single layer (surface) of 2D triangular elements and, ii) it presents the global permeability determined for whole 10-layer laminate. As Figure 6 shows, a good agreement was found between the experimental and numerical results: the infusion took $\approx 1700$ s experimentally while the simulation predicted $1783 \mathrm{~s}$; at $\approx 883 \mathrm{~s}$ the resin flow front advanced approximately the same distance $(\approx 330 \mathrm{~mm})$; and the quantity of resin estimated in the simulation was $723 \mathrm{~g}$, while experimentally $\approx 1520 \mathrm{~g}$ of resin were necessary, due to passive accessories, for a fibre volume fraction of $50 \%$.

a)
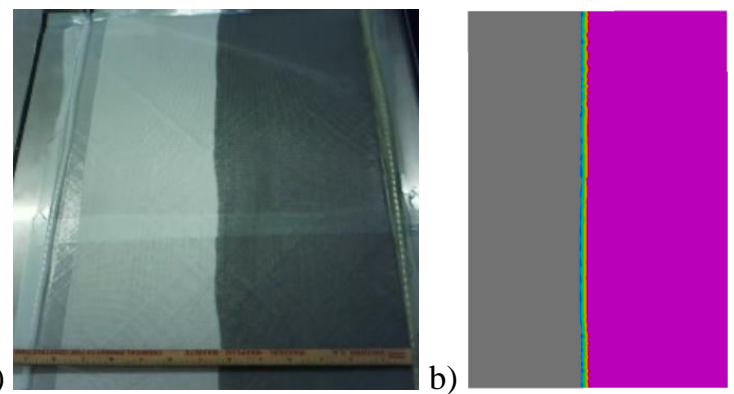

Figure 6- Resin front: experimental (a) and simulation (b) at $t=883 \mathrm{~s}$.

After validation of the laminate properties, the data obtained were used to simulate the vacuum assisted infusion of a component of a car seat (Figure 7-a). The simulation results are presented in Figure 7. During infusion, the resin flows from the region of maximum pressure, at the entry runner, $(1 \mathrm{bar}$, Figure 7-b) toward the region where pressure is minimum (0 bar, Figure 7-b). The distribution of velocity (Figure 7-d) shows that the flow is faster at the concave corners of the geometry than in plane regions and convex corners, as expected and observed experimentally, due to the formation of channels in these regions as a consequence of the reinforcement draping on the geometry. The selected entry and exit ports leaded to a steady progression of the resin flow front along the laminate, and the total impregnation of the laminate is observed, taking 
$2133.3 \mathrm{~s}$ and $\approx 2220$ s to be numerically (Figure 8-c) and experimentally completed, respectively. The good agreement found between the simulations and experimental results shows that the adjustments applied, so far, to the numerical model resulted in a very realistic representation of the infusion of composite parts.

a)
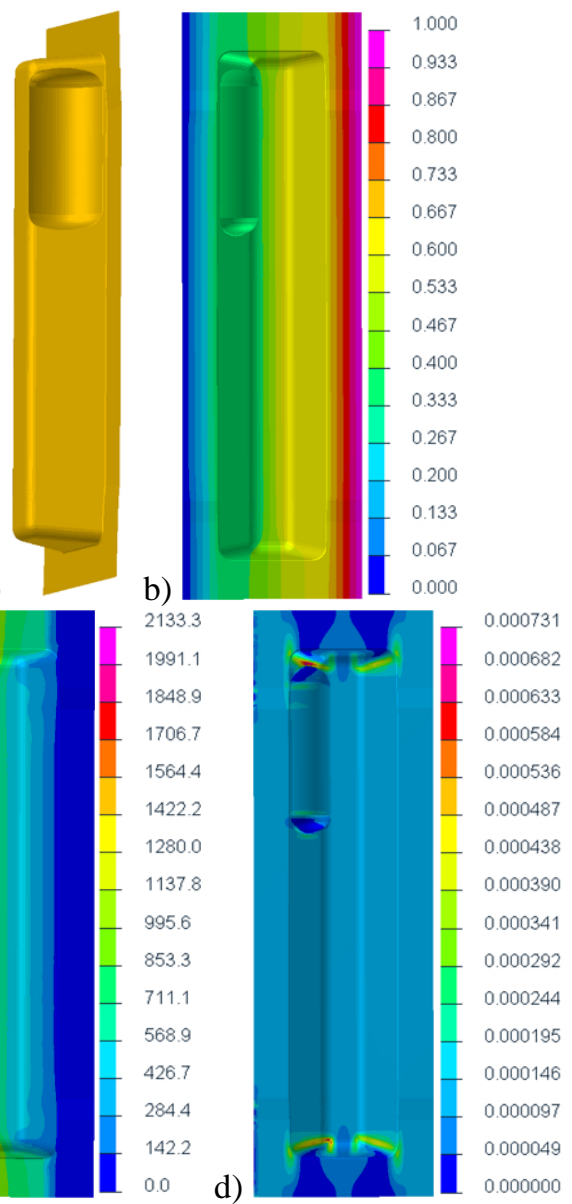

c)

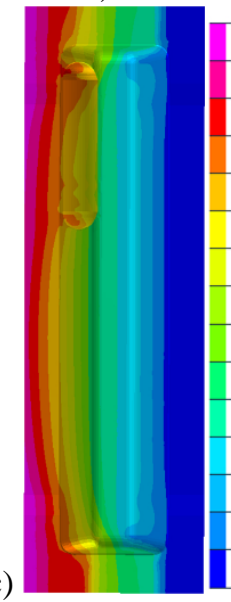

d)

Figure 7-Simulation results of the infusion process of the part of a car seat (a): pressure distribution [bar] (b), filling time [s] (c), flow velocity $[\mathrm{m} / \mathrm{s}](\mathrm{d})$.

\subsection{Sandwich laminates}

Sandwich laminates are used to produce composites requiring both lightweight and high mechanical performances. In general, they are constituted by a core protected by a skin on, at least, two sides of its structure (Figure 8). The adhesion between core and skin is, usually, achieved by the matrix resin, which impregnate both skin and core together. However, in order to get a lightweight composite, the core should not absorb resin within its structure. The adhesion is obtained by particular mechanical finishing, such as, perforations, grooves, gridscores, etc., which guarantee the desired mechanical adhesion (generally designated as biding points).

Thus, along with the simplifications described in the examples above, to simulate the VARI process of a sandwich laminate other assumptions were considered. By way of example, the sandwich laminate depicted in Figure 8 is considered: it uses a perforated PVC foam (core), two plies of biaxial glass fibre fabric on each side (skins) and a polyester resin. The properties of the reinforcements and resin are summarized in Table 4 and Table 2, respectively.

Table 4- Sandwich Laminate - Reinforcement Properties

\begin{tabular}{|c|c|c|}
\hline \multicolumn{3}{|c|}{ Reinforcements } \\
\hline Material & Glass fibre & PVC \\
\hline Density $\left(\mathrm{kg} / \mathrm{m}^{3}\right)$ & 2600 & 100 \\
\hline Structure & biaxial, stitched & foam, perforated \\
\hline Weight $\left(\mathrm{g} / \mathrm{m}^{2}\right)$ & $300 / 300$ & --- \\
\hline Orientation $\left({ }^{\circ}\right)$ & $(612)$ & random \\
\hline Thickness $(\mathrm{mm})$ & $0^{\circ} / 90^{\circ}$ & 10.0 \\
\hline
\end{tabular}

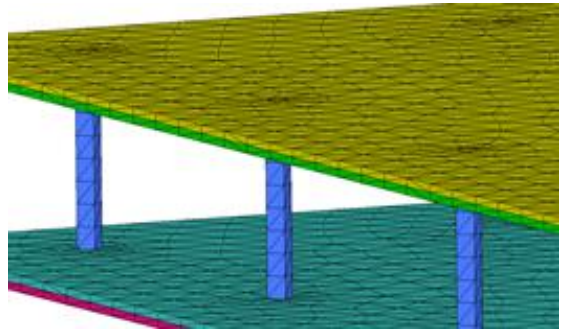

Figure 8- Sandwich laminate structure and mesh.
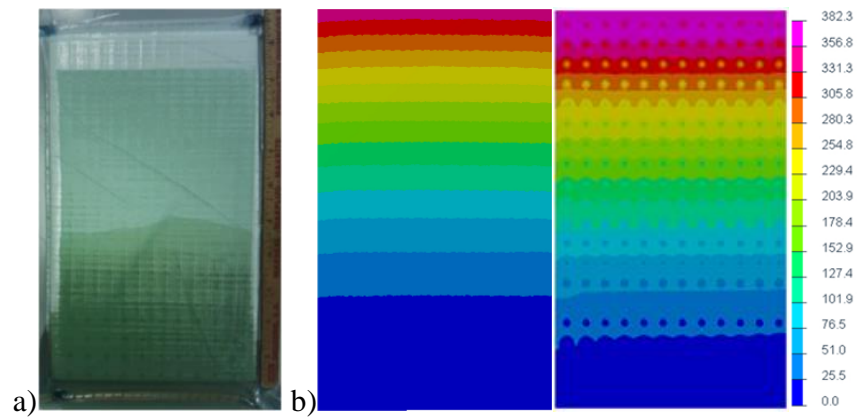

Figure 9- Experimental results: resin front at $\mathrm{t}=60 \mathrm{~s}(\mathrm{a})$; and numerical results: filling time (b).

The main simplifications used to build the mesh were: i) the volume of resin deposited in the surface of the core was neglected and, ii) it was assumed that only the binding points are filled with resin. Thus, instead of building a mesh throughout the volume of the core it was built only at the binding points (in the example, perforations), which guarantee the connection between the core and skins as Figure 8 illustrates. It is admitted that the perforations of the core have maximum permeability in all directions $\left(K_{l}=K_{2}=1.257 \mathrm{E}-05 \mathrm{~m}^{2}\right)$, while the biaxial fabric has $K_{l}=K_{2}=9.913 \mathrm{E}-11 \mathrm{~m}^{2}$, which was determined experimentally as previously described in point 5.1 and Figure 2 by considering a laminate with two biaxial plies.

Following the same previously mentioned method, the material characterization was validated by comparing the numerical and experimental results (Figure 9). Illustrated in Figure 9 is the numerical filling time (of both skins) and the experimental advance of the resin front, at the top skin, in the 
sandwich laminate at $\mathrm{t}=60 \mathrm{~s}$, where good agreement is observed between numerical and experimental results: in both cases, the resin front travelled a distance of $\approx 245 \mathrm{~mm}$ at the top skin, but at the bottom skin the resin front had a delay of $\approx$ $50 \mathrm{~mm}$; while the actual infusion time was $\approx 405 \mathrm{~s}$, numerically was $382 \mathrm{~s}$; and advance of the resin front in the sandwich structure registered was very similar.

\subsection{Hull of a boat}

In the production of composite parts by VARI, in addition to an accurate material characterization, a good definition of the infusion strategy (distribution of resin ports of entry and exit) is mandatory, especially in complex geometries. It is in the definition of the infusion strategy that the simulation of the infusion process has a significant role. Thus, as an example, the steps for the production of a hull boat prototype (Figure 10), with $3 \mathrm{~m}$ length and $1 \mathrm{~m}$ width, are described.

Before simulation, some simplifications were assumed for assuring that an adequate approximation is achieved and, at the
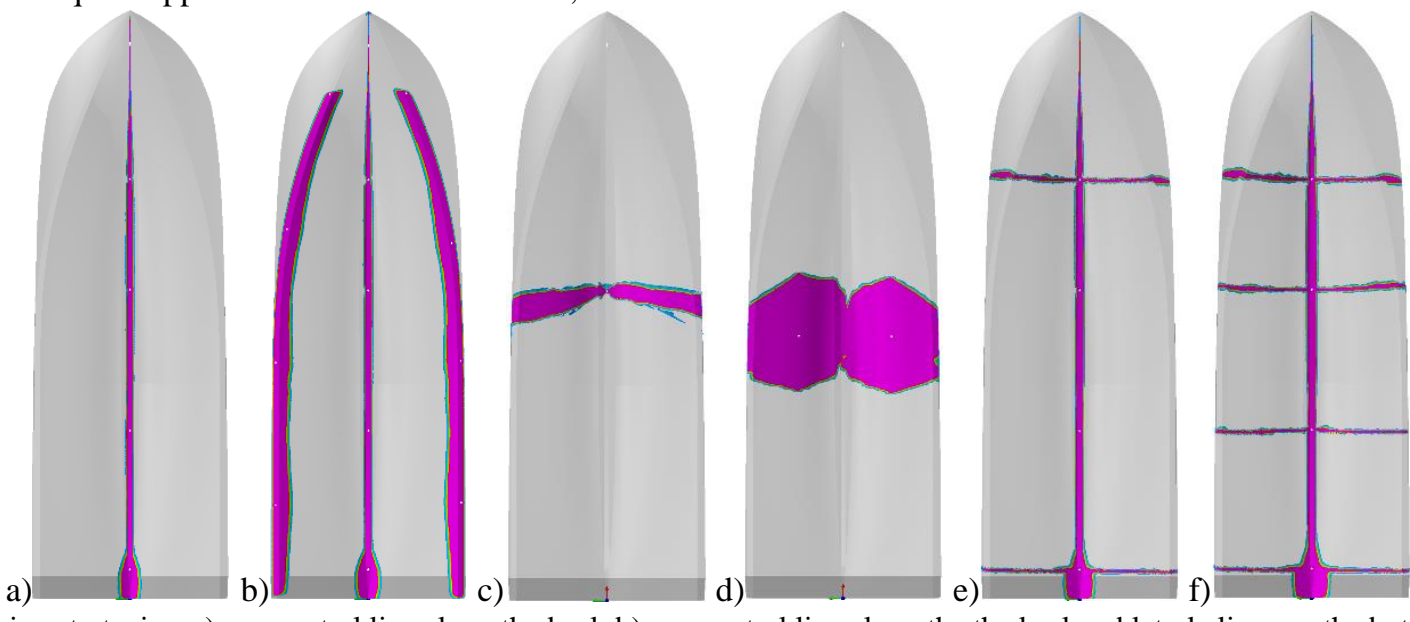

Figure 11- Infusion strategies: a) one central line along the keel; b) one central line along the the keel and laterla lines on the bottom; c) one central entry port on the keel; d) two entry ports on the bottom; e) three lines: one along the keel and two perpendicular at the bow and stern; f) similar to e) but with two more lines at the centre.
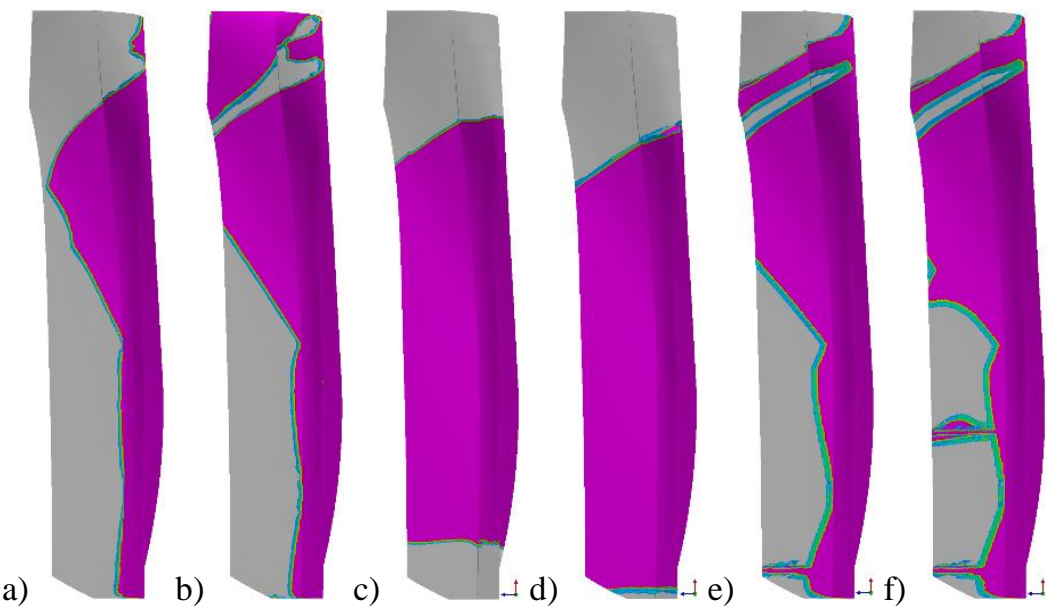

Figure 12- Simulation results: resin front advance for each different infusion strategies illustrated in Figure 11. 
a)
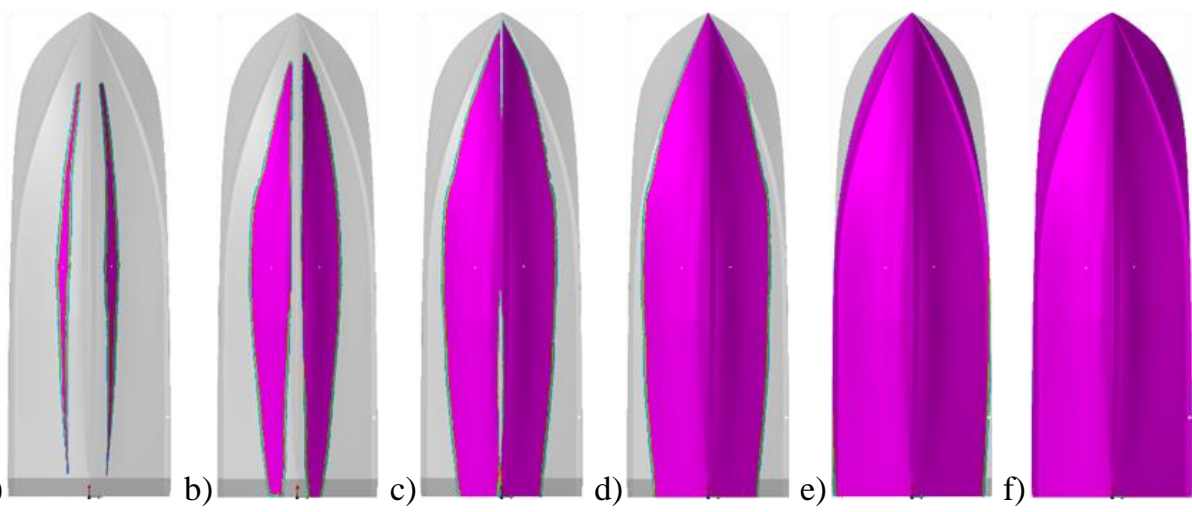

Figure 13- Optimized infusion strategy: resin front advance (filling factor).
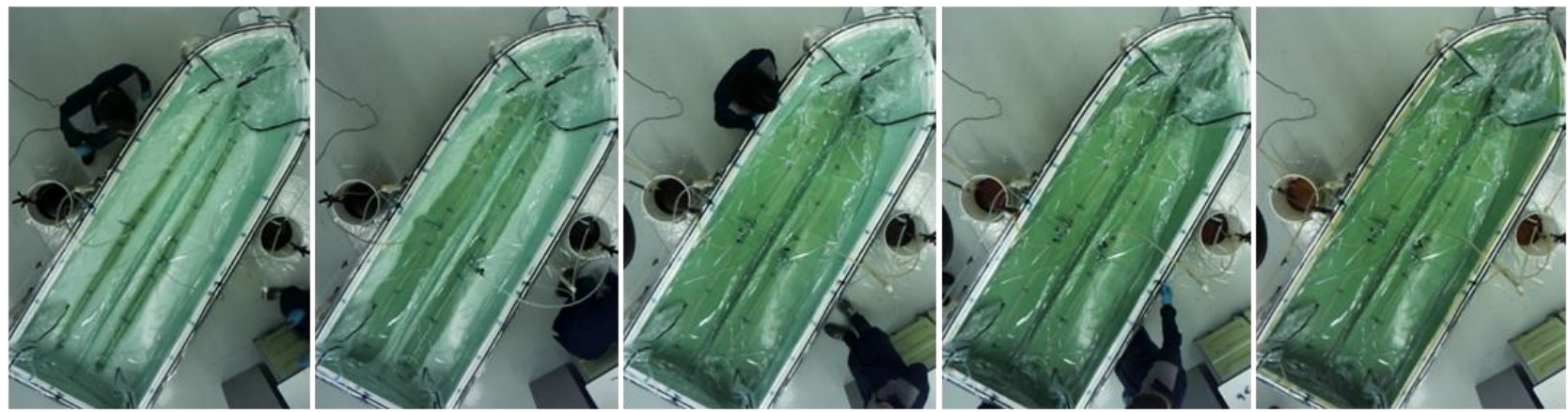

Figure 14- Production of the hull using the infusion strategy illustrated in Figure 13

The matrix was considered to be a polyester resin (Table 2 Table 2), with constant viscosity during infusion, i.e., it has a long gel time and the curing cycle begins only after the complete impregnation of the laminate. The reinforcement is constituted by a sandwich laminate similar to the one depicted in Figure 8, with reinforcement properties described in Table 4. However, instead of a three-dimensional structure (such as the one in Figure 8 and Figure 9), the laminate was considered as an homogeneous material with a global permeability $\left(K_{l}=K_{2}=3.744-09 \mathrm{~m}^{2}\right)$, as in Figure 6 and Figure 7.

After defining the materials parameters, the next step was to define the distribution lines and the entry and exit ports of resin. First, it was stablished that the flow of resin would occur from the bottom of the geometry (keel) to the top (flange) and, so a main exit line was considered along the entire length of the flange. Then, different ports of entry and distribution lines were defined, as Figure 11 shows.

In general, the different infusion strategies revealed not to be adequate to the impregnation of the geometry (Figure 12): regions with high probability of formation of dry zones were detected and not every laminate ended completely impregnated by the resin. Although these observations apply to all cases, significant differences were observed, depending on the infusion strategy: using resin entry ports only (Figure 11- $\mathrm{c}$ and d), the advance of the resin front is uniform and the filling of the laminate is homogeneous (Figure 12- $\mathrm{c}$ and d); using distribution lines (Figure 11- a and b), decreases significantly infusion time but are more likely to form dried regions, particularly at the bow (Figure 12- a and $\mathrm{b}$ ); the addition of perpendicular distribution lines (Figure 11- e and f), results in a decrease of infusion time, but does not decrease the risk of void/dry zones formation (Figure 12- e and f).

Based on these results, the infusion strategy depicted in Figure 14-a was simulated: two distribution lines, ranging from bow to stern, located at the bottom of the hull, arranged parallel to and close to the keel. The distribution lines are opened simultaneously, resulting in the advance of the resin front depicted in Figure 14. The sequence of pictures (Figure 14) show that the laminate was completely impregnated, by a steady resin front. In addition, the flange of the hull is the last region to be impregnated (where the exit line is placed), indicating that the probability to occur voids and dry zones is small. Furthermore, the total infusion time was 6121 seconds (about 102 minutes), which ensures that the infusion takes place within the working time of the resin (Table 2). In the end, the infusion strategy illustrated in Figure 14 revealed to be a good strategy to be applied in the production of the hull.

Using the infusion strategy optimized (Figure 14) the hull of the prototype vessel was produced (Figure 14). Comparing the sequence of pictures from both Figure 13 and Figure 14, it may be observed that the predicted path followed by the resin front mimics accurately the resin flow front advance observed experimentally. Moreover, experimentally, the infusion took about 90 minutes. This difference is justified by the formation of wrinkles, channels and bridges, in the assembly of the laminate and in the sealing of the vacuum bag, where the resin moves at a 
higher speed, compared to the displacement velocity in the laminate. These effects are not considered in the simulation where the laminate arrangement is perfect and the resin only moves faster along boundaries (as in the guitar pickguard) and corners (as in the part of a car seat). Despite the differences, the simulation results reveled to be accurate.

\section{Conclusions}

The simulation program PAM-RTM ${ }^{\circledR}$ from ESI was used in this work to simulate the production of composite parts by using the Vacuum Assisted Resin Infusion (VARI) process. It has shown to be an important tool for predicting the advance of the resin flow front and possible problems and defects that can result from the infusion process.

The accuracy of results is considerably dependent on the material characterisation, particularly, the permeability of the laminate. In order to better compromise the accuracy of results with a reduced simulation time, a global permeability of the laminate should be determined, which considers the combined effect of draping, compression, orientation, etc., and which are difficult to measure. To accomplish that, infusion of the laminate (instead a single layer) to experimentally determine its permeability is, firstly, performed. Then, a simulation of the laminate may be done (admitting that it is a single layer, with the properties of the stack of plies), and the numerical and, finally, the experimental results compared to validate the material characterization. After validation, the infusion process can be simulated for complex geometries with the specific laminate, in order to define the best infusion strategy, predict potential problems and defects, forecast the infusion time, resin quantity, etc. Lastly, the infusion strategy is implemented in the production of a part, so the numerical results can be validated. This method was applied to different laminates (with 1, 5 and 10 plies) and sandwich structures, and used to produce a wide range of parts: from a guitar pickguard to a hull of a vessel.

In addition to the material characterization, several assumptions and simplifications were considered in the simulations in order to obtain more realistic results. It was assumed that the resin behaves like a Newtonian fluid with constant viscosity; presents a sufficiently long gel time and, that the curing process only starts after complete impregnation of the laminate. The mesh is built on the geometry admitting, in most of cases, a 3D tetrahedral elements for the laminate characterization, 2D triangular elements to validate the material properties and to simulate the production of complex geometries; the boundaries of the geometry, regions with sharp concave surfaces or corners, laminate transitions, foam cuts and perforations, etc, are regions with higher velocity flow, compared to that of a laminate. In sandwich structures, it is assumed that the foam does not absorb resin on its surface and within its core, and the resin only flows and fills the biding points.

In general, the simulations were in very good agreement with the experimental results, and the assumptions and simplifications, as in the production of the hull of the boat, helped to increase the accuracy of the results in one hand and, simplified and decreased the simulation time on the other hand.

\section{References}

[1] Dereims, A., Chatel, S.,Marquette, P., Dufort, L. Accurate liquid resin infusion simulation through a fluid-solid couples approach. SAMPE 2017, Seatle, Washington, USA (2017).

[2] Dereims, A., Troian, R., Drapier, S., Bergheau, J-M, de Luca, P. Simulation of liquid resin infusion process by finite element method. ECCM15-15 ${ }^{\text {th }}, 24-28$ June 2012, Venice, Italy (2012).

[3] Dereims, A., Zhao, S., Yu, H., Pasupuleti, P., Doroudian, M., Rogers, W., Aitharaju, V. Compression resin transfer molding (C-RTM) simulation using a coupled Fluid-Solid approach. American Society for Composites $32^{\text {nd }}$ Technical Conference, Indiana, USA (2016).

[4] Pierce, R.S., Falzon, B.G. Simulating Resin Infusion through textile reinforcement materials for the manufacture of complex composite structures. Engineering 3, 596-607 (2017).

[5] PAM-RTM 2013: User's Guide \& Tutorials, Esi Group.

[6] Zhao, C., Zhang, G., Wu, Y. Resin flow behaviour simulation of grooved foam sandwich composites with vacuum assisted resin infusion (VARI) moulding process. Materials 5, 1285-1296 (2012).

[7] Celle, P., Drapier, S., Bergheau, J.-M. Numerical modelling of liquid infusion into fibrous media undergoing compaction. European Journal of Mechanics A/Solids 27, 647-667 (2008).

[8] Yang, B., Jin, T., Li, J., Bi, F. Simulation prediction of the preform deformation and resin vacuum infusion process. Polymer Composites, 1968-1979 (2014).

[9] Dereims, A., Drapier, S., Bergheau, J-M, de Luca. 3D robust iterative coupling of Stokes, Darcy and solid mechanics for low permeability media undergoing finite strains. ESI-Technical Paper, 1-50, November (2014). 